\title{
Black Hole Entropy, Verlinde's entropy gravity proposal and Unruh formula in Projection Gravitation
}

\author{
Author: XiaoLin Li (Chongqin, China), hidebrain@hotmail.com \\ Corresponding author: XiaoLin Li (Chongqin, China), hidebrain@hotmail.com
}

\begin{abstract}
In the projection gravitation theory, exist a merging model of black hole formation. Particles that meet the merging conditions can be combined together to form a particle system. This particle system, as a whole, manifests itself as a black hole. The essence of a black hole is an empty hole. A black hole is not a single entity, it is a composite particle system. From this model, the black hole entropy formula (actually the empty hole entropy) can be easily derived out. This combined condition is equivalent to a temperature condition. From this black hole model, we can easily derive out the Verlinde's entropy gravity proposal formula, and the Unruh formula can be easily deduced. It is also proved that the Verlinde's entropy gravity proposal formula and the Unruh formula are actually equivalent. Projection gravitation is a reasonable quantum gravitation theory, which has important research value.
\end{abstract}

\section{Keywords}

projection gravitation, black hole, empty hole, empty hole entropy, empty hole temperature, black hole entropy, black hole temperature, merging conditions, temperature condition, the principle of uncertainty, Verlinde's entropy gravity proposal, Unruh formula, quantum gravitation.

\section{Introduction}

In the author's previous paper[2], the author discusses the merging model of black hole formation and deduces the black hole entropy formula. In this paper, the authors continue to discuss the property of this black hole model, revealing more surprising conclusions. From this black hole model, the Verlinde's entropy gravity proposal formula can be derived out, and the Unruh formula can be derived out. Through these discussions, human understanding of gravitation and black hole entropy can be further improved. About the connection between gravitation and thermodynamics, it requires further reflection.

For readers who don't know the merging model of black hole in projection gravitation, please read the author's previous paper[2] first. It is only after understanding the merging model of black hole in projection gravitation that we can understand what is discussed in this paper.

Please note that unless other specifically stated, the following discussion of gravitation and black holes refers to the objects in projection gravitation theory. Unlike other theory of gravitation and black holes, there are differences. Please take care to distinguish and not to confuse.

\section{Black hole entropy in projection gravitation}


In the author's previous paper[2], the author take a conclusion. The black hole at the center of gravitation is actually an empty hole. External particles, attracted by the central black hole, are infinitely close to the black hole, but cannot fall into the black hole. There is a distance limit between particles and black holes. This distance limit is the radius of a black hole. So, to be precise, a black hole is an empty hole in projection gravitation. External particles, if met with a special condition, can be close to the radius of the black hole, and can be merged together with the black hole to form a larger black hole. Through this model, the black hole entropy formula and the black hole temperature formula can be derived out very simply. This black hole entropy formula, has only one constant difference between the Beckenstein and Hawking black hole entropy formula. The formula form is exactly the same.

The formula for the total number of particles in a black hole is (1.1). $r$ is the radius of a black hole.

$$
\mathrm{N}=\frac{c^{3}}{2 \hbar G} r^{2}
$$

The total energy formula of a black hole is (1.2). $r$ is the radius of a black hole.

$$
\mathrm{E}=\frac{C^{4}}{G} r
$$

The entropy formula of a black hole is (1.3). $r$ is the radius of a black hole. $n$ is the number of degrees of freedom of the particles that make up the black hole.

$$
\mathrm{S}=\frac{k_{B} C^{3} \ln n}{2 \hbar G} r^{2}
$$

The temperature formula of a black hole is (1.4). $r$ is the radius of a black hole. $n$ is the number of degrees of freedom of the particles that make up the black hole.

$$
\mathrm{T}=\frac{\hbar c}{\ln n k_{B} r}
$$

Here, the author corrects two errors in the previous paper and apologizes to the reader.

(1). To the black hole merging model in the previous paper stated, there were inaccuracies and uncertainties. It's not two black holes that actually merge together. The correct statement is that. The center is a black hole and the other is actually a particle near the black hole. The mass of this near-particle is $m_{0}$, which meets the condition of (5.1) in the previous paper. Repeat this process, so it can be concluded that the most central particle is the original seed of the black hole. The original black hole seed at this center is the real singleparticle black hole. The particle system, which these particles combine to form, as a whole, for the outside, is a black hole. Entering the inside of this black hole, the particles inside the black hole are not black holes, just ordinary particles. This combination of black holes is a composite object. This kind of composite black hole is a thermodynamic system with black hole entropy and black hole temperature. This special condition (5.1) is met between particles in this black hole system. This particular physical system, as a whole, for the outside, is a system with the nature of a black hole.

However, in the projection gravitation theory, every particle with rest mass has the property of black hole radius. So, in this sense, it can also be thought of as a merging of two black holes. However, it is important to distinguish precisely the similarities and differences between concepts. Particles with rest mass of $m_{0}$ in the process of merging have the inherent length property of black hole radius, but are not real black hole in themselves. 
So, in projection gravitation, a black hole is actually a particle system. This particle system, as a whole, for the outside, manifests itself as a black hole. However, the individual particles that make up this black hole are not, in fact, black holes in themselves. There are great differences between the concept of black holes in projection gravitation, the concept of black holes in other gravitation theory. Please note the difference here.

(2). In the previous paper, the author calculated that the black hole temperature formula was $\mathrm{T}=\frac{2 \hbar c}{\ln n k_{B} r}$ (5.10 in previous paper). There is a calculation error here, and the correct result is $\mathrm{T}=\frac{\hbar c}{\ln n k_{B} r}$.

This composite black hole model in projection gravitation is a physical system in the thermodynamic sense, with internal structure, internal distribution, and is a true thermodynamic black hole. This kind of black hole, which has entropy, has a reasonable origin of physics.

In the discussion of the merging process of black hole formation, the author obtains the merging condition (1.5) of the formation of a black hole based on the quantum mechanical equation of the gravitation field. Only two particles that meet this condition can be combined to form a system of black holes.

$$
m_{0} C^{2}=\frac{\hbar C}{r}
$$

The specific physical significance of the condition (1.5) was not carefully elaborated in the previous paper. So, in this paper, the authors continue to discuss this merging process of black hole formation.

In the process of merging this black hole, there should be a special particle at the center of the black hole, the central particle, which is the original seed of the black hole. This primitive seed, which attracts a condition-satisfying (1.5) particle close, merges together to form a system of black holes. In this way, the black hole is growing, forming a large black hole.

For ease of description, use a new constant symbol, $D$, to identify the constant of $\ln n$. Please pay attention to it.

$$
\mathrm{D}=\ln n
$$

So can get (1.7). This is the energy equalization theorem of black holes.

$\mathrm{E}=2 \mathrm{DN} k_{B} T$

It is not $\mathrm{E}=\mathrm{N} k_{B} T / 2$. Why there are differences in constants, this topic needs further study.

In fact, for (1.5), the more general case would be as (1.8). The numerical change of constant value $\xi$ only changes the value of the constant in the derivation result and does not change the physical nature of the theory. The exact value of the constant value $\xi$ needs to solve the exact solution of the equation of the projection gravitation field wave function in order to be determined. Also, there may be different types of particles, and the $\xi$ values of the particles may be different.

$$
m_{0} C^{2}=\frac{\xi \hbar C}{r}
$$

\section{Temperature condition in merging process}


As shown in Figure 1, there have two particles. One is A. Its rest mass is M. $r$ is its radius of black hole, $\mathrm{r}=\frac{G M}{C^{2}}$. The other is $\mathrm{B}$, rest mass is $m_{0}$. The two objects merge together to form a black hole.

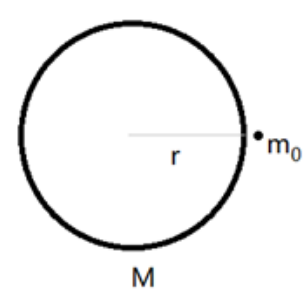

Figure 1

According to the merging model of black hole formation in the previous paper, particle B must meet the condition, the formula (2.1).

$$
m_{0} C^{2}=\frac{\hbar C}{r}
$$

What is the physical significance of this formula? This formula means that. If there is a particle $A$ whose black hole radius is $r$. If there is another particle $B$, can merge with $A$ to form a black hole $C$. The rest mass of the particle B must meet the formula of (2.1). Particles of other rest mass cannot be combined with particle $A$ to form a black hole. This is the merging condition of black hole formation.

Please note that in the projection gravitation, particle has a black hole radius as long as it has rest mass. The radius of this black hole is actually a empty hole radius. The distance of other particles, and the center of the particle, can only reach this distance at a minimum. Therefore, the radius $r$ of the black hole is also an inherent property of the particle. This is the difference between projection gravitation and other gravitation. When particle $B$ is near the black hole radius of particle $A$, for $B$, particle $A$ is a black hole. So, in this merging process, particle $A$ can also be thought of as a black hole. But particle $A$ is not a black hole for other particles that are not close to $A$. Please note the difference here. This is a very special point in the projection gravitation. Any particle with rest mass has an inherent black hole radius. The radius of a black hole is a distance property of a particle.

Condition (2.1) is derived out from the quantum mechanical wave function equation of projection gravitation. Only if this condition is met, the particle $B$ in the gravitation field of $A$, only in the lowest energy, it is possible to merge with $A$. This condition, from the point of view of quantum mechanics, is an obvious condition. Two particles are merged, and it is clear that the two particles should have the greatest chance of getting close to each other, and the most likely to merge. $B$ is at the lowest energy level of the A gravitation field, the probability of being close to $A$ is the greatest, and the probability of a merger occurring is the greatest. This is a very obvious result. From the quantum energy formula of projection gravitation, it can be seen that when particle $B$ is at the lowest energy in particle A gravitation field, the rest mass of $B$ meets the condition (2.1). This is the physical significance of (2.1) in quantum mechanics. This condition is actually the result of the calculation of quantum mechanical equations in projection gravitation.

In thermodynamics, the temperature of particle B satisfies (2.2). 
$\mathrm{E}=\frac{1}{2} k_{B} T$

So

$\mathrm{E}=\frac{1}{2} k_{B} T=m_{0} C^{2}=\frac{\hbar C}{r}$

So

$\mathrm{T}=\frac{2 \hbar C}{k_{B} r}$

(2.3) is the temperature of particle $B$.

The temperature of the black hole $A$ is (1.4).

The temperature of particle $B$, and the temperature of black hole $A$, has only one difference in proportional constant. About go constant, roughly speaking, can be considered as temperature equality.

Therefore, from the thermodynamic point of view, the merging process of black hole formation, there is a temperature condition. Two objects must meet this temperature condition in order to merge to form a black hole. Two objects that do not meet this temperature condition cannot merge to form a black hole.

Condition (2.1) is equivalent to a temperature condition.

It is also possible to describe the merger process of the formation of this black hole. Particle B, incorporated into a black hole A, forms a new black hole C. Particle B must meet this temperature condition in order to fit into black hole $A$. Otherwise, particle B cannot be integrated into black hole A. Therefore, for a black hole, only external particles that meet this special temperature condition can be integrated into the black hole.

From the quantum mechanical wave function equation of projection gravitation, there is an energy condition in the process of merging. From the thermodynamics, there is a temperature condition in the process of merging. There is a wonderful correlation between the two. This may be the physical origin of gravity with a wonderful thermodynamic nature.

We approximately think that the particle momentum is $\mathrm{P}=m_{0} C, \mathrm{E}=\mathrm{PC}=$ $m_{0} C^{2}$. Particles are close to the black hole, and the shortest distance is the black hole radius r. From the principle of uncertainty, approximately is $\mathrm{p}=\frac{\hbar}{c}$, so get $m_{0} C^{2}=\frac{\hbar c}{r}$. It is the condition (2.1)。So, from the principle of uncertainty, can derive out the condition (2.1). (2.1) is equivalent to a temperature condition. So, we can think so. In quantum gravitation, there is a wonderful correlation between the uncertainty principle and the temperature of thermodynamics. Temperature is correlated with entropy. Entropy and uncertainty are related The principle of uncertainty is a sign of uncertainty. Therefore, the author boldly speculates that the uncertainty principle may be the real physical source of entropy. This topic is worthy of further study.

We can also find that the coefficient $\xi$ change in (1.8) only changes the proportional value between the temperature of the two objects. It does not change the essence of a fixed proportional relationship between the temperature of the two objects.

\section{Derive out Verlinde's entropy gravity proposal formula}

Continuing the discussion above, in this merging process, let's calculate the entropy of 
particle B.

To particle $B$, it satisfies $\mathrm{T}=\frac{d E}{d S}$ 。 So get

$\frac{d S}{d r}=\frac{1}{T} \frac{d E}{d r}$

Because the energy of particle B satisfies $\mathrm{E}=m_{0} C^{2}$. And it satisfies (2.1). So get

$\frac{d E}{d r}=\frac{d\left(\frac{\hbar C}{r}\right)}{d r}=-\frac{\hbar c}{r^{2}}$

In merging process, particle B satisfies the temperature condition. So the temperature of particle $B$ is (3.3).

$$
\mathrm{T}=\frac{2 \hbar C}{k_{B} r}
$$

Take (3.2) and (3.3) into (3.1), so get

$\frac{d S}{d r}=-\frac{k_{B} r}{2 \hbar C} \frac{\hbar C}{r^{2}}=-\frac{k_{B}}{2 r}$

From (2.1), can get $r=\frac{\hbar}{m_{0} c}$. Take it into (3.4), so get

$\frac{d S}{d r}=-\frac{k_{B}}{2 \hbar} m_{0} C$

This is the change of the entropy of particle B during the merging process. The negative sign indicates the direction of the gravitational pull of particle B. The change of entropy is caused by gravitation. Therefore, this change of entropy reflects the nature of the gravitational pull of particle $B$.

As you can see, in addition to the differences in constants, the form of the formula (3.5) is exactly the same as Verlinde's entropy gravity proposal formula.

The key of the whole derivation process lies in the existence of temperature condition (2.1). So, as you can see from here, Verlinde's entropy gravity proposal formula is actually the process by which particles merge into a black hole, or, in other words, a particle into a black hole. Verlinde's entropy gravity proposal formula can be derived out in the merging process of black hole formation. So Verlinde's entropy gravity proposal formula is just an export of projection gravitation.

Verlinde's entropy gravity proposal formula is equivalent to the temperature condition (2.1).

In fact, the theory of entropy gravity hypothesis is very similar to the merging process of the formation of black holes in projection gravitation. The gravity of entropy is a particle incorporated into a virtual holographic screen, the radius of the holographic screen increases. Projection gravitation is a combination of particles and a black hole to form a new black hole, with an increased radius of the black hole.

But in fact, in the projection gravitation, particles have always been particles, and black holes have always been black holes. just two objects close together, from the outside, it seems that the formation of a new black hole, or, the radius of the black hole is increasing. Merge, just close to the distance, and not really blend in. Integration is only an observation from an outside perspective.

In this process of merging, it can be seen that only particles that meet the condition (2.1) 
can be integrated into the black hole. Other particles cannot be integrated into a black hole. But the theory of entropy gravity hypothesis assumes that all rest mass particles can be integrated into a holographic screen. Therefore, from the derivation in projection gravitation, the accuracy of the assumption of entropy gravity, there is doubt, it is questionable.

From the discussion of the merger process above, it can be seen that the holographic principle and the entropy of black holes in projection gravitation are exactly corresponding. Holographic screens can be thought of as virtual black holes. In projection gravitation, a black hole is actually an empty hole, and not a single entity, but a system of particles. This particle system, on the whole, for the outside world, shows the nature of a black hole, with black hole entropy, with black hole temperature. A black hole in projection gravitation is not a bottomless abyss of the general relativity that inhales all matter. The empty hole model in the projection gravitation is actually more in line with the concept of holographic screen.

\section{Derive out Unruh formula}

Let's continue to discuss the merging process.

The temperature of particle $B$ is (2.3).

To the black hole $A$, it satisfies

$\mathrm{r}=\frac{G M}{C^{2}}$

Take (4.1) into (2.3), get

$k_{B} \mathrm{~T}=\frac{2 \hbar C}{r}=\frac{2 \hbar C r}{r^{2}}=\frac{2 \hbar G M}{C r^{2}}$

$\frac{G M}{r^{2}}$ is the gravitational acceleration a on the surface of the black hole A. So get

$k_{B} T=\frac{2 \hbar a}{C}$

In addition to the differences in constants, (4.3) is exactly the same as Unruh formula.

So, we derive out the results of the Unruh formula from the process of merging. Therefore, it can be seen that the Unruh formula is only a manifestation of the nature of this merging process.

In fact, the Unruh formula can also be derived out directly from the black hole entropy. Therefore, it can be concluded that the Unruh formula is just another expression of the black hole entropy formula.

The temperature of the black hole is (1.4), so get

$k_{B} \mathrm{~T}=\frac{\hbar C}{D r}=\frac{\hbar C r}{D r^{2}}$

Because $\mathrm{r}=\frac{G M}{C^{2}}$, so

$k_{B} \mathrm{~T}=\frac{\hbar C r}{D r^{2}}=\frac{\hbar C r}{D r^{2}} \frac{G M}{C^{2}}=\frac{\hbar}{D C} \frac{G M}{r^{2}}=\frac{\hbar a}{D C}$

It is only one constant difference from (4.3).

Therefore, we can see that the Verlinde's entropy gravity proposal formula and Unruh 'formula, in fact, are the nature of merging process of the black hole formation, both of which are actually equivalent. Gravitation can no longer be derived out using the Verlinde's entropy 
gravity proposal formula and the Unruh formula. Verlinde's entropy gravity proposal is, in practice, circular reasoning.

gravitational acceleration a on the surface of the black hole, $\mathrm{a}=\frac{G M}{r^{2}}$. And $\mathrm{r}=\frac{G M}{C^{2}}$. We change form of the formula. We can get a very surprising result.

$$
\mathrm{a}=\frac{G M}{r^{2}}=\frac{1}{r} \frac{G M}{r}=\frac{C^{2}}{r}
$$

The gravitational acceleration on the surface of a black hole is a very simple relationship between the radius of the black hole. (4.6) and (1.4), both of which take very similar forms. Therefore, it is clear that the black hole temperature, and the surface acceleration of the black hole, there is a simple proportional relationship. This is actually the result of derivation from int the projection gravitation theory. To the Unruh formula, there is no mystery.

\section{Conclusion}

In the above discussion, the author discusses in detail the merging process of black hole formation in projection gravitation theory. The surprising results are derived out. In the merging process, we derive out the Verlinde's entropy gravity proposal formula, and we derive out the Unruh formula. It is also found that the Verlinde's entropy gravity proposal formula and the Unruh formula are actually equivalent, both of which are the same physical source, both from the merging process which form the black hole.

We also found that in the quantum mechanical equations of projection gravity field, there is a curious correlation between the particle energy and the thermodynamic temperature, between the uncertainty principle and the thermodynamic temperature. This association is the physical origin of gravitation, which has a very special thermodynamic nature. This topic is well worth studying.

The projection gravitation theory can derive out many meaningful physical results. It is a reasonable quantum gravitation theory, and has important research value. The authors expect more researchers to join the study of projection gravitation theory, revealing more properties of gravitation.

\section{References}

[1] XiaoLin Li.

Projection Gravitation, a Projection Force from 5dimensional Space-time into 4dimensional Space-time International Journal of Physics , 2017, 5(5), 181-196. DOI: 10.12691/ijp-5-5-6

http://pubs.sciepub.com/ijp/5/5/6/index.html.

[2] XiaoLin Li.

Empty Hole Entropy and Black Hole Entropy in Gravitation

International Journal of Physics, 2018, 6(5), 155-160. DOI: 10.12691/ijp-6-5-3.

http://pubs.sciepub.com/ijp/6/5/3/index.html.

[3] Bekenstein,Jacob D. (April 1973).

Black holes and entropy

Physical Review D 7(8): 2333-2346. 
[4] Bardeen,J. M.; Carter, B.; Hawking, S. W.

The four laws of black hole mechanics

Comm.Math. Phys. 31 (1973), no. 2, 161-170.

[5] E. P. Verlinde

On the Origin of Gravity and the Laws of Newton

JHEP 1104 (2011) 029 [arXiv:1001.0785 [hep-th]].

[6] G. 't Hooft

Dimensional reduction in quantum gravity

arXiv:gr-qc/9310026.

[7] W. G. Unruh

Notes on black hole evaporation

Phys. Rev. D 14, 870 (1976).

[8] David Chandler

introduction to modern statistical mechanics

ISBN-13: 978-0195042771.

[9] R. P. Feynman,R. B. Leighton,M. Sands

The Feynman Lectures on Physics (Volume I,II,III)

ISBN 9787506272476,ISBN 9787506272483,ISBN 9787506272490. 